\title{
El gasto en la inversión pública y su impacto en la reducción de la pobreza monetaria en el Perú. Periodo 2000-2018
}

\section{Spending on public investment and its impact on the reduction of monetary poverty in Peru. Period 2000-2018}

\author{
Eduardo Quiroz Vera ${ }^{1 *}$ \\ ${ }^{1}$ Facultad de Ingeniería Económica, Estadística y Ciencias Sociales, Universidad Nacional de Ingeniería, \\ Lima, Perú \\ *E-mail: equiroz@uni.edu.pe \\ Recibido (Received): 23/06/2020 Aceptado (Accepted): 07/10/2020 Publicado (Published): 15/12/2020
}

\begin{abstract}
RESUMEN
El objetivo de la presente investigación fue analizar el impacto del gasto en inversión pública sobre la reducción de la pobreza monetaria en el Perú para el periodo 2000-2018. La investigación es cuantitativa, de tipo descriptivo y explicativo, de diseño correlacional y de corte longitudinal. Para cuantificar los efectos de la inversión pública sobre la pobreza, se utilizó información secundaria de fuentes como las series de tiempo del Banco Central de Reserva del Perú y estadísticas del Banco de Proyectos del Ministerio de Economía y Finanzas y del Instituto Nacional de Estadística e Informática. Los resultados indican que el gasto público en inversión y los niveles de pobreza poseen una relación inversa, tanto a nivel total, como a nivel de los sectores que conforman la inversión pública ejecutada. Se concluye que la gestión de la inversión pública logró reducir la pobreza monetaria en el Perú a lo largo del periodo de estudio, siendo solo la inversión en el sector social la que ha tenido una participación significativa en la reducción de la pobreza.
\end{abstract}

Palabras Clave: Inversión pública, niveles de pobreza, pobreza monetaria

\begin{abstract}
The objective of this research was to analyze the impact of public investment spending on the reduction of monetary poverty in Peru for the period 2000-2018, the research is descriptive and explanatory, of correlational design, of longitudinal cut, to quantify the Effects of public investment on poverty, secondary information was used from sources such as the time series of the Central Reserve Bank of Peru and statistics from the Project Bank of the Ministry of Economy and Finance and the National Institute of Statistics and Informatics, the results indicate that public investment spending and poverty levels have an inverse relationship both at the total level and at the level of the sectors that make up the executed public investment, it is concluded that the management of public investment managed to reduce monetary poverty in Peru throughout the study period, being only investment in the social sector that has had a participation significant in reducing poverty.
\end{abstract}

Keywords: Public investment, poverty levels, monetary poverty 


\section{INTRODUCCIÓN}

Erradicar la pobreza en todas sus formas sigue siendo uno de los principales desafíos que enfrenta la humanidad. Si bien la cantidad de personas que viven en la extrema pobreza disminuyó en más de la mitad entre 1990 y 2018, aún demasiadas luchan por satisfacer las necesidades más básicas.

Diversos organismos, como el Programa de las Naciones Unidas para el Desarrollo, el Banco Mundial o el Fondo Monetario Internacional, han establecido diferentes formas de medición para la pobreza. Por otro lado, cada país realiza estadísticas propias para evaluar sus niveles de pobreza; en la presente investigación, se definirá la pobreza desde la perspectiva de la pobreza monetaria.

Para el Programa de las Naciones Unidas para el Desarrollo (PNUD), en la actualidad, la pobreza es multidimensional y abarca mucho más que el ingreso. La pobreza multidimensional captura la realidad de vivir con menos de lo necesario para llevar una vida digna. Unos 1300 millones de personas viven en pobreza multidimensional, y la mitad son menores de 18 años, según el Índice Multidimensional de Pobreza (IPM) de 2018 publicado por el Programa de las Naciones Unidas para el Desarrollo (PNUD) y la Iniciativa de Oxford sobre Pobreza y Desarrollo Humano (OPHI, por sus siglas en inglés).

El Índice, que analiza 105 países y abarca casi las tres cuartas partes de la población mundial, mira más allá de los ingresos para comprender cómo las personas experimentan la pobreza de manera múltiple y simultánea. Identifica cómo las personas están quedando rezagadas en tres dimensiones clave: salud, educación y nivel de vida, incluyendo 10 indicadores, entre ellos la falta de acceso a agua potable, nutrición adecuada y educación primaria. Las personas que experimentan privación en al menos un tercio de estos indicadores ponderados entran en la categoría de pobreza multidimensional.

A nivel mundial, alrededor de 736 millones de personas aún viven con menos de US\$1,90 al día (2015) y muchos carecen de acceso a alimentos, agua potable y saneamiento adecuados. El crecimiento económico acelerado de países como China e India ha sacado a millones de personas de la pobreza, pero el progreso ha sido disparejo. La posibilidad de que las mujeres vivan en situación de pobreza es desproporcionadamente alta en relación con los hombres, debido al acceso desigual al trabajo remunerado, la educación y la propiedad.

Los avances también han sido limitados en otras regiones, como Asia Meridional y África subsahariana, donde vive el $80 \%$ de la población mundial que se encuentra en condiciones de extrema pobreza. Además, nuevas amenazas que plantean el cambio climático, los conflictos y la inseguridad alimentaria necesitan mayores esfuerzos para sacar a las personas de la pobreza. (PNUD). 
Según la CEPAL (Comisión Económica para América Latina y el Caribe), en 2017, el número de personas que vivían en la pobreza llegó a 184 millones (30,2\% de la población), de los cuales 62 millones se encontraban en la extrema pobreza (10,2\% de la población, el porcentaje más alto desde 2008). Sin embargo, la proporción de personas en situación de pobreza extrema continuó creciendo, siguiendo la tendencia observada desde 2015.

El INEI (Instituto Nacional de Estadística e Informática) considera como pobres monetarios a las personas que residen en hogares cuyo gasto per cápita es insuficiente para adquirir una canasta básica de alimentos y no alimentos (vivienda, vestido, educación, salud, transporte, etc.). Son pobres extremos aquellas personas que integran hogares cuyos gastos per cápita están por debajo del costo de la canasta básica de alimentos. Al término del año 2018 , el $20,5 \%$ de la población del país - que equivale en cifras absolutas a 6593000 personas - se encontraba en situación de pobreza; es decir, tenían un nivel de gasto inferior al costo de la canasta básica de consumo compuesto por alimentos y no alimentos. La tasa de pobreza del 2018 reflejó una disminución de 1,2 puntos porcentuales en comparación con el resultado en 2017. Con esto, unas 313000 personas salieron de la pobreza monetaria el año pasado.

El Perú ha avanzado significativamente en su crecimiento económico en los últimos 15 años. Dicho crecimiento económico ha ido acompañado por un aumento sostenido del gasto público en general y, en este contexto, se obtuvo una reducción significativa niveles de pobreza. Desde el 2004, según Instituto Nacional de Estadística e Informática (INEI) la pobreza total cayó del 58,7\% al 20,5\% en 2018.

Según el Banco Mundial (2017), los logros económicos y sociales en el Perú fueron posibles en un contexto internacional, caracterizado por el incremento del precio de los minerales, componente principal de las exportaciones del Perú. El éxito económico en el periodo comprendido entre los años 2006 y 2015 se expresa en el crecimiento del Producto Bruto Interno (PBI), en un promedio anual del 6\%; así como una acertada política económica y al incremento de los precios de exportación de los minerales en el mercado internacional. Esto contribuyó de manera significativa para que el Perú ejecute los más altos niveles de inversión pública, en comparación con sus pares latinoamericanos y con los países de la Organización de Cooperación y Desarrollo Económico (OCDE). En términos absolutos, en el periodo comprendido entre el año 2000 y 2007, el gasto público en inversiones fue de S/ 30756 120; este monto se multiplicó por 5,6 veces para el periodo comprendido entre 2008 y 2018.

En el Perú, la situación de la población en pobreza se mantuvo superior al $50 \%$ durante toda la década del 90. En el periodo objeto de estudio, entre los años 2000 y 2004, en promedio, el nivel de pobreza tiende a crecer y toca su valor máximo en el año 2004, con el $58,7 \%$ de la población peruana en situación de pobreza. Es a partir del año 2005 que los 
niveles de pobreza en el Perú tienden a disminuir de manera sostenida, reduciéndose para el año 2018 al 20,5\% de la población del país. En trece años, la pobreza monetaria se redujo en 38,2 puntos porcentuales. No obstante, la pobreza rural sigue siendo alta con el $42,1 \%$ de pobreza, siendo tres veces más que en las zonas urbanas $(14,4 \%)$.

En junio de 2000, se creó la Ley № 27293 del SNIP (Sistema Nacional de Inversión Pública), con el objeto de "optimizar el uso de los Recursos Públicos destinados a la inversión, mediante el establecimiento de principios, procesos, metodologías y normas técnicas relacionados con las diversas fases de los proyectos de inversión". Para febrero del 2017, entró en vigencia el Sistema Nacional de Programación Multianual y Gestión de Inversiones, creado con Decreto legislativo 1252, cuya finalidad es "orientar el uso de los recursos públicos destinados a la inversión para la efectiva prestación de servicios y la provisión de la infraestructura necesaria para el desarrollo del país" (MEF, 2016).

Haciendo un análisis de los resultados del SNIP, podemos citar lo siguiente: En el 2005 el gobierno nacional ejecutó en inversión pública un monto de S/. 2756 millones de nuevos soles (1 751 proyectos declarados viables), creciendo al 2010 a S/. 5087 millones (1 841 proyectos declarados viables) y, finalmente, llega al 2015 con una ejecución de S/. 27319 millones de nuevos soles (1 751 proyectos declarados viables). Los gobiernos regionales ejecutaron en el 2005 S/. 1342 millones de nuevos soles (1 342 proyectos declarados viables), subiendo al 2010 a S/. 6480 millones (1 289 proyectos declarados viables) y, posteriormente, alcanza al 2015 con una ejecución de S/. 9539 millones de nuevos soles (1 091 proyectos declarados viables).

La ejecución de los gobiernos locales en el 2005 fue de S/. 726 millones de nuevos soles (923 proyectos declarados viables), llegando al 2010 a S/. 9539 millones (10 470 proyectos declarados viables) y alcanzado al 2015 la ejecución de S/. 9539 millones de nuevos soles (19 942 proyectos declarados viables).

Existen investigaciones que indican la existencia de una relación directa entre el gasto público en infraestructura y la reducción del nivel de pobreza. A nivel internacional, podemos citar a Chotia y Rao (2017), quienes investigaron sobre si el desarrollo de infraestructura puede contribuir en la reducción de la pobreza en Brasil, Rusia, India, China y Sudáfrica en el periodo 1991-2014. Los resultados obtenidos confirman que existe evidencia de causalidad a corto plazo que va desde el crecimiento económico y el desarrollo de infraestructura hasta la reducción de la pobreza; además, concluyen que el desarrollo de la infraestructura ayuda en reducir la pobreza. En esa misma línea, Ali y Pernía (2003) concluyen que la inversión en infraestructura vial, eléctrica, de riego y, en general, infraestructura social y productiva, constituyen medios que permiten mejorar los ingresos de los habitantes de las zonas de influencia de dichos proyectos, al expandir sus fronteras productivas, facilitar acceso a mercados, aumentar productividad y generar ingresos por mano de obra, lo cual contribuye a reducir los niveles de pobreza. 
En el Perú, se han realizado pocos trabajos de investigación relacionados con la medición de los impactos de la inversión en infraestructura básica y la reducción de la pobreza. Alvarado (2018), en su investigación sobre el Análisis de la gestión del gasto público en inversión y su incidencia sobre la reducción de los niveles de pobreza en el Perú, encontró que la gestión de la inversión pública logró reducir la pobreza. A lo largo del periodo de estudio, la gestión del gasto público en inversión y los niveles de pobreza poseen una relación inversa. En esa misma línea, Vilca (2018), en su trabajo de investigación titulado Inversión pública y su relación con los niveles de pobreza monetaria en las regiones del Perú: periodo 2004-2015, corroboró la hipótesis planteada de que existe una relación lineal inversa entre la inversión pública y la pobreza monetaria en las regiones del Perú comprendidas entre los años 2004-2015. Es decir, a mayores niveles de inversión pública menores niveles de pobreza monetaria. Por otro lado, a nivel de regiones, hay investigaciones realizadas en el tema como el de Huaquisto (2018), quien estudió la inversión pública y pobreza monetaria en el departamento de Puno: periodo 2004-2015, encontrando que las inversiones públicas tienen efectos positivos (estimado con signo negativo y significativo) en la disminución de la pobreza monetaria, ya que en promedio por cada 100 millones de soles que se ejecute en un período anterior en proyectos de inversión pública en el departamento de Puno, el indicador de pobreza se reducirá en 4,45\%; también encontró evidencia de que la inversión pública social es la que más ha contribuido en reducir la pobreza. Así mismo, Rojas (2016) encontró que la pobreza en la región Amazonas ha tenido una disminución relativa. En la provincia de Condorcanqui, se muestra una tasa de pobreza en el año 2009 de 83,3\%, mientras que, en el año 2013, según Endes, con un intervalo de confianza del 95\%, aparece con $69,9 \%$ y $76 \%$. También, Fort y Paredes (2015), en su investigación sobre la Inversión pública y descentralización: sus efectos sobre la pobreza rural en la última década, muestran que los componentes de riego, caminos, telecomunicaciones y programas de apoyo al productor tienen un efecto significativo sobre la reducción de la pobreza rural, y que este se manifiesta principalmente por medio de mejoras en la productividad agrícola. Hallaron también que el fortalecimiento del capital humano y las inversiones en conectividad y acceso a mercados también resultan relevantes, con un impacto que se manifiesta mediante cambios en los ingresos y la composición de la ocupación rural.

Esta realidad sobre el crecimiento sostenido del gasto en Inversión Pública y su impacto en la reducción de los niveles de pobreza es nueva en la historia de la economía y de la sociedad peruana, motivo por el cual es necesario conocer cuantitativamente este resultado. En ese contexto, el objetivo de la investigación es Analizar el impacto del gasto en inversión pública sobre la reducción de la pobreza monetaria en el Perú para el periodo 2000-2018, con la intención de contribuir al debate académico, en la perspectiva de un gasto público más eficiente. Así mismo, en relación con el desarrollo teórico precedente, la hipótesis de la investigación quedó formulada de la siguiente manera: El gasto en Inversión Pública ejecutada tiene una relación directa e inversa con el nivel de pobreza en el Perú, periodo 2000-2018. 


\section{MATERIALES Y MÉTODOS}

La investigación tiene un enfoque cuantitativo, de tipo descriptivo y explicativo. Descriptivo, en tanto que se establecen los patrones del nivel de pobreza y del gasto en inversión pública mediante gráficos. Explicativo, en razón de que se busca conocer la relación del gasto en inversiones, según sectores públicos, sobre el nivel de la pobreza monetaria en el Perú en el periodo 2000-2018. Se ha utilizado como fuente las series de tiempo del Banco Central de Reserva del Perú y estadísticas del Banco de Proyectos del Ministerio de Economía y Finanzas y del Instituto Nacional de Estadística e Informática.

El procesamiento de los indicadores se realizó a través del Software estadístico SPSS; también se hizo uso de Excel para el análisis gráfico de las tendencias de las variables. Para el análisis econométrico, tendiente a explicar la relación existente entre la gestión del gasto Público y los niveles de pobreza en el Perú en el periodo de 2000 al 2018, se especificó una relación de variables de la siguiente manera:

Inversión pública $t \Rightarrow$ pobreza monetaria $t$

Donde el subíndice $t$ denota el tiempo en que es tomado el dato.

Matemáticamente la función es así:

pobreza monetaria $t=($ inversión pública $t)$

Donde el comportamiento de la tasa de pobreza monetaria en el tiempo t depende de la inversión pública realizada en el tiempo t.

El modelo econométrico de panel de datos se especifica así:

tasa de pobreza monetaria $t=i n v e r s i o ́ n ~ p u ́ b l i c a ~ t+X t+\varepsilon t$

Donde $X t$ son otras variables dependientes del modelo y $\varepsilon t$ es un término de perturbación estocástica.

Para suavizar los cambios bruscos de la variable inversión pública se lo transforma a términos per cápitas y en logaritmos para aproximar los datos a una curva normal, además los parámetros estimados se interpretan como la variación promedio de las variables.

Los modelos a estimar son:

Modelo 1

Log.pobreza $=\beta 0+\beta 1 \log$. (inv.pub) $+\mu t$

Modelo 2

log.pobreza $=\beta 0+\beta 1 \log$.inv.sec. eco. $+\beta 2$ log.inv.sec. soc. +

$\beta 3$ log.inv.sec. gen. $+\beta 4$ log.inv.pro. mul. $+\mu t$ 
Donde:

log.pobreza: logaritmo tasa de pobreza monetaria.

log.inv.pub: logaritmo de inversión pública.

log.inv.pub.sec. eco.: logaritmo de la inversión pública en los sectores económicos.

log.inv.sec. soc.: logaritmo de la inversión pública en los sectores sociales.

log.inv.sec. gen.: logaritmo de la inversión pública en los sectores generales

log.inv.pro. mul.: logaritmo de la inversión pública en programas multisectoriales

Tanto para el modelo 1 y 2 se usan los métodos de efectos fijos y efectos aleatorios. Además, para darle más eficiencia a los modelos y para controlar los problemas de heterocedasticidad y autocorrelación, los modelos se estimaron por mínimos cuadrados generalizados factibles.

\section{RESULTADOS}

\subsection{ANÁLISIS GRÁFICO DE LAS VARIABLES DE STUDIO}

A continuación, para cada una de las variables del estudio se hará una descripción gráfica de su tendencia y comportamiento en el periodo de análisis.

\subsection{LA POBREZA MONETARIA}

La figura 1 describe cómo ha evolucionado la pobreza monetaria para el periodo 20002018. Se observa que, como consecuencia del segundo gobierno del ingeniero Alberto Fujimori, que terminó en medio de una profunda crisis política, lo llevó a la contracción de la economía y la reducción de la inversión pública y privada, que agravaría la pobreza del 2000 (50,1\%) hasta el 2004 (58,7\%). A partir del 2005, la pobreza se va reduciendo y, a finales del 2006, la pobreza fue del 49,2\%, lo cual se debió a que en el gobierno de Toledo, el gasto en proyectos y programas sociales representó más del $25 \%$ del presupuesto público. Para el segundo gobierno de Alan García, la pobreza se redujo de $42,4 \%$ (2007) a $27,8 \%$ (2011). El gasto público en inversión en proyectos y programas sociales se enfocó las zonas rurales y lo que dinamizó programas como "Juntos, Beca 18, Pensión 65", etc., lo que contribuyó a la reducción de la pobreza. Al término del Gobierno de Ollanta Humala, en el año 2016, la economía creció en 4,9\%, mientras que la pobreza alcanzó al 20,7\% de la población. La pobreza monetaria se redujo en el Perú a $20,5 \%$ en el 2018 , desde un nivel previo de $21,7 \%$ en el 2017 , lo que implica que 313000 peruanos abandonaron esa situación el año pasado. 
Figura 1. Perú: Evolución de la pobreza monetaria total 2000-2018

(Porcentaje respecto del total de la población)

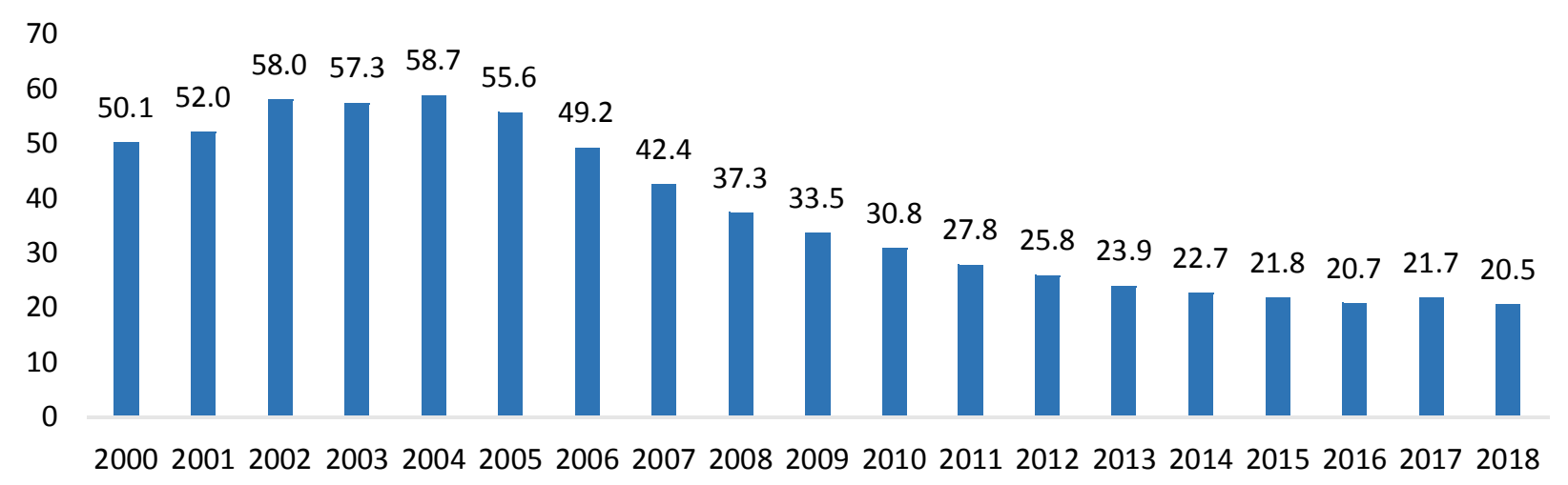

Fuente: INEI

\subsection{INVERSIÓN PÚBLICA}

En la Figura 2, la Inversión Pública ejecutada que en el 2000 fue de S/ 4762 millones y para el 2018 alcanzó a un monto de S/.21 004 millones; es decir, en 19 años se incrementó en $441,1 \%$. Los sectores que componen la Inversión Pública son: (a) El Sector Económico (que comprende la inversión en Agricultura, Transportes, Energía, Industria, Comercio, Turismo y Pesquería) que poseen el siguiente comportamiento, en el año de 2000 era de S/. 2 346,1 millones, en el año 2018, aumentó a S/.10 035,7 millones (428\%); (b) El Sector social (que es el Gasto Público ejecutado en proyectos de educación, salud, trabajo y otros de ámbito social), que en 2000 fue de $\mathrm{S} / .1533,5$ millones y se incrementa en el 2018 a S/. 6 790,6 millones (443\%); (c) Los Sectores Generales (que comprende el Gasto en Inversión de proyectos de inversión de la Presidencia del Consejo de Ministros, Ministerios de Justicia, Interior, Relaciones Exteriores, Economía y Finanzas, Ministerio Público y Jurado Nacional de Elecciones) poseen similar comportamiento de crecimiento en el año 2000 alcanzaba el monto de inversión de $S / .434 .5$ millones, aumentó a $S / .3$ 177,9 millones (731\%); (d) por último, los Programas multisectoriales (que incluyen proyectos de inversión del ámbito sectorial diverso) posee el siguiente comportamiento para el año 2000: la formación bruta de capital fijo fue de $\mathrm{S} / .447,8$ millones que se incrementa al año 2018 a $\mathrm{S} / .999,8$ millones (223\%). 
Figura 2. Perú: Evolución de la Inversión Pública 2000 - 2018

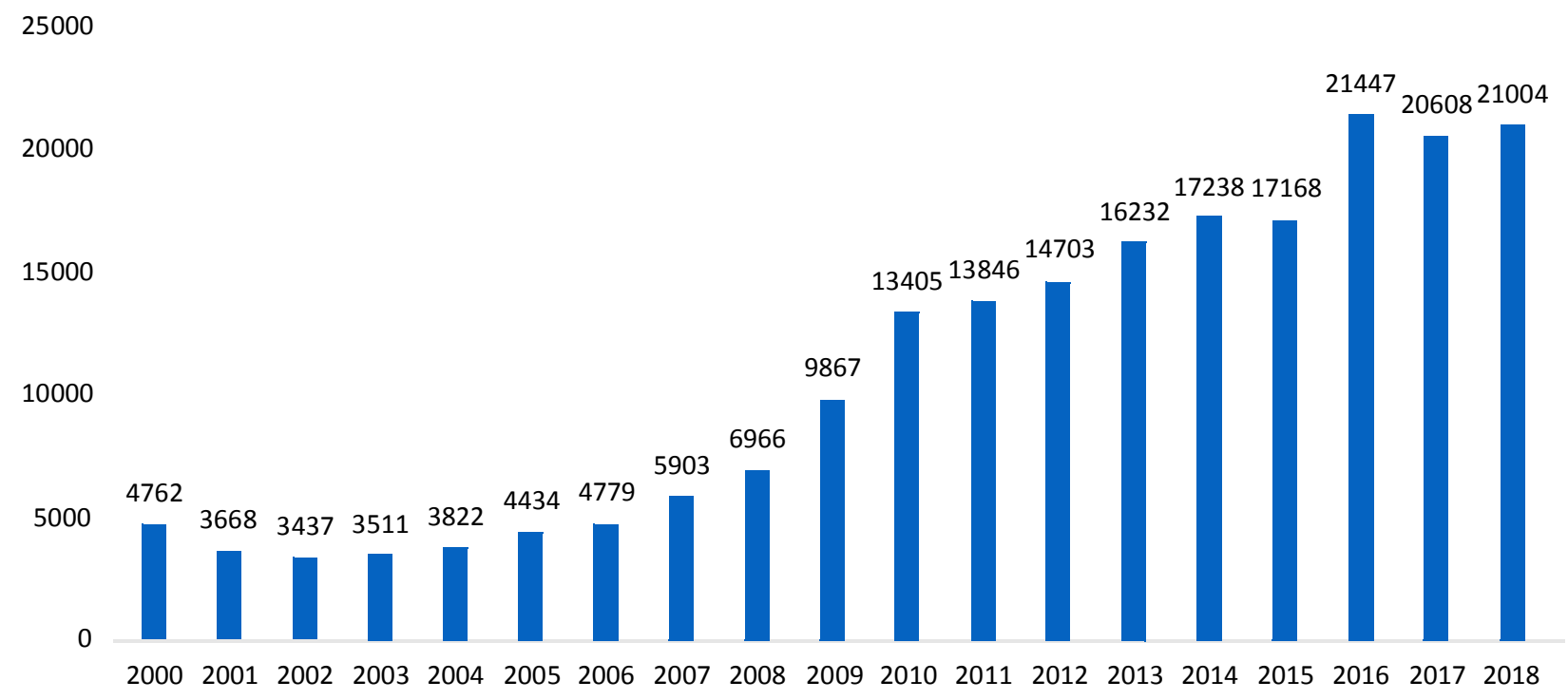

Fuente: Banco Central de Reserva del Perú

La crisis política del año 2001 hizo que el Estado disminuya su participación en la formación del producto de la economía. Esta crisis afectó hasta el 2003, y a partir de allí hay un moderado avance en inversión pública hasta el 2006 (S/.4 779 millones). Pero, a partir del 2007, hay un acentuado crecimiento del gasto público en inversión que pasó de S/.5 903 millones hasta S/.17 238 millones en el 2014. Esto debido a que la economía peruana creció encima del 5\%, mayor inversión privada, alta capacidad de gasto del Gobierno proveniente de impuestos de canon y regalías mineras. El continuo crecimiento de la inversión pública es al 2018 de S/.21 004 millones.

\subsection{RELACIÓN ENTRE LOS NIVELES DE POBREZA Y EL CRECIMIENTO DE LA INVERSIÓN PÚBLICA}

La figura 3 presenta en términos generales una relación inversa entre los Niveles de pobreza monetaria y el crecimiento de la Inversión Pública, ya que un mayor volumen de inversión pública lleva consigo un efecto inmediato de mayor decrecimiento de los niveles de pobreza. En el periodo del 2000 al 2004, la dinámica de las variables de estudio fue muy baja, por ejemplo en el año 2000 , el 50,1\% de la población era pobre, contando con una inversión pública de S/.4 762 millones, cinco años después, las cifras de pobreza llegan a $58,7 \%$ (2004) y de inversión del sector público a 3822 millones (2004); pero, a partir del 2005, la evolución de la inversión pública va en crecimiento, de S/.4 454 millones (2005), pasa al 2010 a 13405 millones y, en 2016, llega al monto de S/. 21447 millones, afectando a la pobreza en 55,6\% (2005), 30,8\% (2010) y 20,77\% (2016). En el 2017, la Inversión pública baja a S/. 20608 millones debido a que se frenó las operaciones vinculadas a casos de corrupción, y para el 2018 sube a S/. 21004 millones, en contraste con la pobreza en 21,7\% 
(2017) y 20,5\% (2018). En consecuencia, desde el 2005, la gestión inversión pública ha tenido un impacto significativo.

Figura 3: Perú: Niveles de Pobreza VS Gasto en Inversión Pública. 2000 - 2018 (VAR\%).

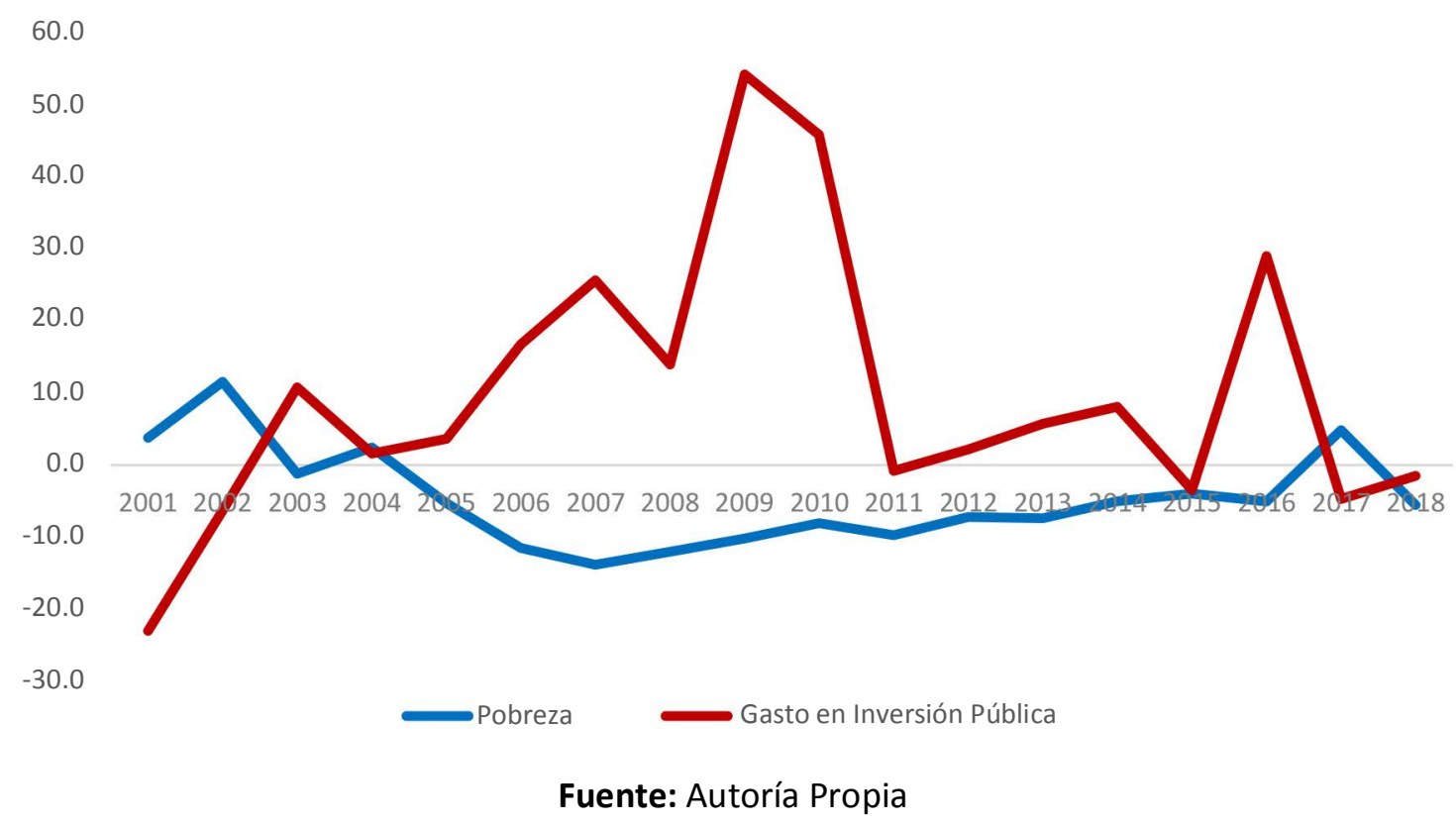

\subsection{ANÁLISIS ECONOMÉTRICO}

En la figura 4 se representa el diagrama de dispersión entre el logaritmo de la tasa de pobreza monetaria y el logaritmo del gasto en la Inversión Pública que muestra una relación lineal inversa entre ambas variables.

Figura 4: Gráfico de dispersión entre los Niveles de pobreza y el gasto en Inversión Pública

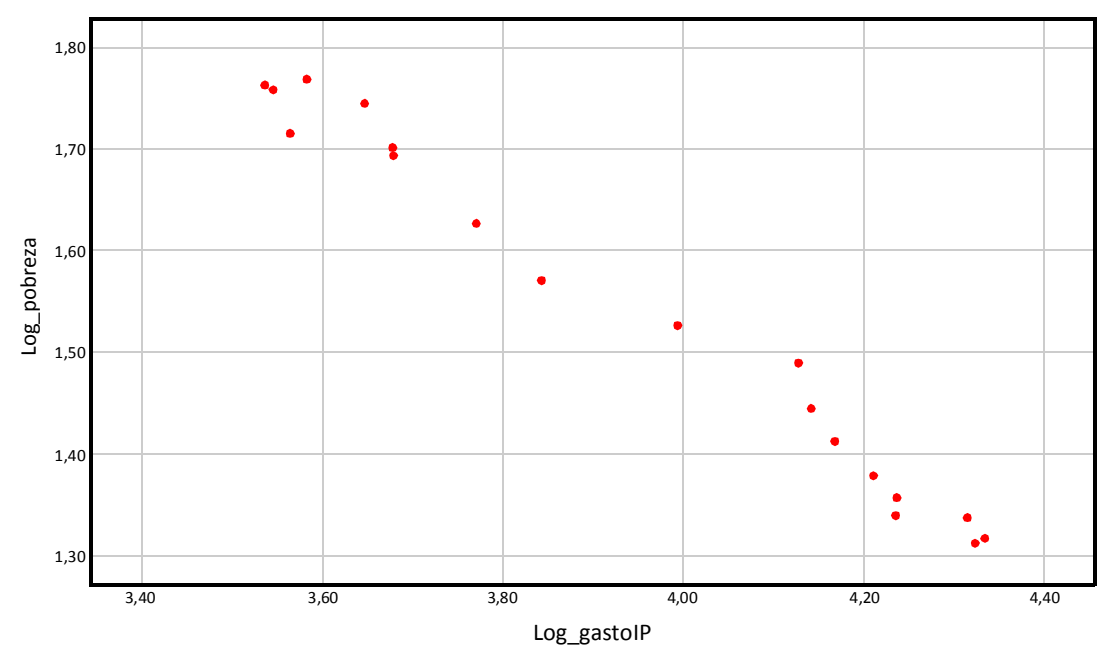

Fuente: Autoría propia. 


\subsection{MODELOS ECONOMÉTRICOS}

Se ha considerado 2 modelos econométricos para ver el efecto del gasto en la inversión pública ejecutada sobre la tasa de pobreza monetaria. En la tabla 1 se resume las estimaciones de los parámetros de las variables dependientes de los dos modelos y, además, se muestran los niveles de significancia y el R-squares que mide la bondad de ajuste de los modelos econométricos.

\section{Modelo 1}

$\log$ pobreza $=\beta 0+\beta 1 \log$ (gasto en la inversión pública) $+\mu 1 t$

Se incluyó solo la variable gasto en la inversión pública en los logaritmos con el fin de observar el efecto de esta variable en el logaritmo de la tasa de pobreza monetaria. Los parámetros estimados por los métodos de efectos fijos y mínimos cuadrados generalizados factibles son significativos $(p<0,05)$. Además, $\beta 1$ tiene el signo esperado, el efecto es de reducción de la tasa de pobreza monetaria.

Por otro lado, dado que las variables en los modelos están en logaritmos tanto dependientes como independientes, la estimación de los parámetros se interpreta con la elasticidad de la tasa de pobreza monetaria dado un cambio del $1 \%$ en la variable Formación Bruta de Capital Fijo Público. El parámetro estimado $\beta 1=-0,571$ significa que al aumentar en $1 \%$ la inversión pública, la pobreza monetaria se reduciría en $0,571 \%$.

Según el valor del coeficiente de determinación, R2 $=0,983$, revela que hay un alto grado de correlación entre las variables pobreza e Inversión pública; es decir, las variaciones de la pobreza están siendo explicadas en un 98,3\% por variaciones en la Inversión pública.

El valor de la prueba de Durbin Watson $=1,346$ nos indica la incorrelación de los residuos. Para descartar el fenómeno de regresión espuria o regresión sin sentido, se ha aplicado la regla estadística desarrollada por Granger y Newbold (citado en Gujarati y Porter, 2010). Esta regla establece que si el coeficiente de determinación (R2) es mayor al estadístico " $d$ " Durbin-Watson se concluye que la regresión es espuria o regresión sin sentido; sin embargo cuando sucede lo contrario $(R 2<d)$, se descarta tal fenómeno. Para ambos modelos se ha encontrado que R2 <d, por tanto, las regresiones estimadas no son espurias. Dicho esto, todas las pruebas estadísticas aplicadas a la regresión de largo plazo estimada son válidas y coherentes. 
Tabla 1: Resultados del efecto de la inversión pública sobre la pobreza.

\begin{tabular}{|c|c|c|c|c|}
\hline \multirow{2}{*}{ Variable } & \multicolumn{2}{|c|}{ Modelo 1} & \multicolumn{2}{|c|}{ Modelo 2} \\
\hline & Coef. & $\mathbf{t}$ & Coef. & $\mathbf{t}$ \\
\hline \multirow[t]{2}{*}{ C } & 3.792 & 52.117 & 3.482 & 22.624 \\
\hline & $(0.000)$ & & $(0.000)$ & \\
\hline \multirow[t]{2}{*}{ Log FBKIP } & -0.571 & -31.146 & & \\
\hline & $(0.000)$ & & & \\
\hline \multirow[t]{2}{*}{ Log Sector Económico } & & & -0.123 & -0.811 \\
\hline & & & $(0.431)$ & \\
\hline \multirow[t]{2}{*}{ Log Sector Social } & & & -0.329 & -2.242 \\
\hline & & & $(0.042)$ & \\
\hline \multirow[t]{2}{*}{ Log Sector Generales } & & & -0.070 & -0.691 \\
\hline & & & $(0.501)$ & \\
\hline \multirow[t]{2}{*}{ Log Sector Prog. Multisectoriales } & & & -0.055 & -0.965 \\
\hline & & & $(0.351)$ & \\
\hline $\mathrm{R}^{2}$ & 0.983 & & 0.985 & \\
\hline $\mathrm{R}^{2}$ Ajustado & 0.982 & & 0.980 & \\
\hline \multirow[t]{2}{*}{ F estadístico } & 963.853 & & 226.742 & \\
\hline & $(0.000)$ & & $(0.000)$ & \\
\hline D.W. & 1.346 & & 1.547 & \\
\hline
\end{tabular}

Fuente: Autoría propia.

\section{Modelo 2}

log.pobreza $=\beta 0+\beta 1 \log$.sec.econ.$+\beta 2 \operatorname{logsec}$. soc. $+\beta 3$ log.sec.general + $\beta 4 \log$. sec.prog.multisec $+\mu$ it

Este modelo contempla incluir en la regresión a la inversión pública en sus componentes, esto es la inversión en el sector económico, sector social, sectores generales y sector de programas multisectoriales; todas esas variables en términos per cápita y en logaritmos. Los métodos de estimación son los ya mencionados en el modelo anterior y la interpretación de los coeficientes es el de la elasticidad.

Con respecto a los componentes de la inversión pública, todas tienen una elasticidad menor a cero y solo la inversión en el sector social es estadísticamente significativa en la reducción de la pobreza $(p<=0,05)$, mientras que las otras no son estadísticamente significativas $(p>0,05)$. Sin embargo, los resultados de la prueba $F$ indican que de forma conjunta los coeficientes de las cuatro variables explicativas son estadísticamente significativos y distintos de cero, ya que esta prueba $\mathrm{F}$ tiene un valor de 226, 742. Por tanto, todas las variables deben ser consideradas en el modelo para explicar el comportamiento de la pobreza monetaria. 
El parámetro estimado $\beta 2=-0,329$ significa que al aumentar en $1 \%$ la inversión pública, la pobreza monetaria se reduciría en 0,329\%.

Para este modelo, valor del coeficiente de determinación, $\mathrm{R} 2=0,985$ revela que hay un alto grado de correlación entre las variables pobreza e Inversión pública, es decir, las variaciones de la pobreza están siendo explicadas en un 98,5\% por variaciones en la Inversión pública en los sectores económicos, social, generales y programas multisectoriales.

El valor de la prueba de Durbin Watson $=1,547$ nos indica la incorrelación de los residuos.

Los modelos econométricos estimados son una representación simplificada de la relación entre el gasto en inversión pública ejecutada y nivel de pobreza para el periodo 2000-2018, cuyo uso permite hacer predicciones del valor futuro del nivel de pobreza, como consecuencia de las variaciones del gasto público. Una forma de evaluar el impacto de los proyectos de inversión en los niveles de pobreza sería la aplicación de estos modelos econométricos en los procesos de programación multianual de inversiones (PMI). En el sector público peruano, facilitaría la evaluación del impacto de los proyectos de inversión en los niveles de pobreza, mediante criterios técnicos en lugar de procedimientos preferentemente políticos.

\section{DISCUSIÓN DE RESULTADOS}

Los modelos estimados dan de manifiesto la importancia que tiene la inversión pública en el proceso de reducción de pobreza a nivel nacional (es decir, mayor gestión de inversión pública se reduce los niveles de pobreza en el periodo 2000-2018), acorde con la tesis de Quiñones (2016), aunque en su variable dependiente asume al gasto público total, esta tiene el efecto de hacer caer la tasa de pobreza monetaria ante un incremento del gasto público total. Igualmente, la investigación de Fort \& Paredes (2014) encuentra que invertir en infraestructura pública rural como canales de regadío o programas de apoyo al productor tiene un efecto significativo en la reducción de la tasa de pobreza rural. En ese sentido, esta investigación corrobora la hipótesis planteada de que existe una relación lineal inversa entre los niveles de inversión pública y los niveles de pobreza monetaria en el Perú, periodo 2000-2018. Los resultados para el sector social sí contribuyen a la reducción de la pobreza, por cada incremento de $1 \%$ en la formación bruta de capital fijo público en este sector se ha logrado que los niveles de pobreza se reduzcan en 0,0329\%. Ponce (2013) concluye "la relación entre inversión pública y PBI es positiva y significativa, inversión privada, superficie agrícola y capital humano, la inversión pública ha resultado ser un factor relevante en el crecimiento económico". De hecho, por los resultados obtenidos se puede afirmar que inversión pública sí incide en la reducción de los Niveles de Pobreza. En esa misma línea, Briones y Peña (2018) concluyen que la inversión en programas sociales contribuyó positivamente al desarrollo social; esto medido como IDH. 
Por otro lado, también se corrobora el enfoque de activos de la pobreza, en el que el Estado juega un rol importante como gestor de igualdad de oportunidades, así, la inversión pública permite mejorar la competitividad; por ejemplo: de productores rurales, al tener una carretera que permite tener acceso a mercados y mejorar sus costos de transacción. Una parte complementaria al servicio de la educación es la construcción de escuelas y colegios para que más niñas y niños tengan acceso a la educación pública. También necesaria es la inversión en infraestructura de salud. Tener postas, puestos de salud y hospitales tiene un impacto sustancial en mejorar las condiciones de vida de la población. Por otro lado, la inversión pública es fuente generadora de empleo, al ser esta intensiva en mano de obra poco calificada que al final se traducen en ingresos para los hogares más vulnerables.

Este trabajo expone el desempeño del Estado a nivel de políticas de inversión y concluye que el Estado sí tuvo un mediano desempeño en sus funciones respecto de la lucha contra la pobreza.

\section{CONCLUSIONES}

- Durante el periodo de análisis, del 2000 a 2018, la tasa de pobreza monetaria se redujo en 37 puntos porcentuales, es decir de 50,1\% en el 2000 a 20,5\% en el 2018 . Entre 2004 y 2015, durante un periodo de fuerte y sostenido crecimiento macroeconómico, la incidencia de la pobreza en el país se redujo en 36,9 puntos porcentuales. Pese a la desaceleración económica que afecta a la región en los últimos años, el Perú ha continuado reduciendo la pobreza. De hecho, en 2018, el Perú creció $4,0 \%$ y la pobreza se redujo 1,2 puntos porcentuales, mientras que en América Latina el crecimiento fue de 1,3\% y la reducción apenas alcanzó 0,1 puntos porcentuales".

- Durante el periodo de estudio, las inversiones públicas nacionales crecieron de S/ 4762 millones (2000) hasta S/.21 004 millones (2018); es decir, en 19 años se incrementó en $441,1 \%$. El crecimiento de los sectores que componen la Inversión Pública en el periodo de referencia fue de la siguiente manera: El Sector Económico (428\%); El Sector social (443\%); los Sectores Generales (731\%); por último, los Programas multisectoriales (223\%).

- Los modelos econométricos estimados permiten corroborar la hipótesis planteada de que existe una relación lineal inversa entre los niveles de inversión pública y los niveles de pobreza monetaria en el Perú, periodo 2000-2018. Para la estimación del modelo 1 , se encontró que al aumentar en $1 \%$ la inversión pública, la pobreza monetaria se reduciría en 0,571\%; mientras que en el modelo 2, para el caso de los sectores que componen la inversión pública, es decir: la inversión pública en el sector económico, sector social, sectores generales y los programas multisectoriales, las elasticidades son menores a cero y solo la inversión en el sector social es estadísticamente significativa, y en los otros sectores no se encuentra coeficientes significativos. 


\section{RECOMENDACIONES}

Se recomienda al Gobierno Central aumentar la inversión pública en el sector social, ya que, como se evidenció en esta investigación, la inversión pública en este sector tiene un efecto en la reducción de la tasa de pobreza monetaria; así mismo, a los Gobernadores Regionales y a los alcaldes provinciales y distritales, reforzar y elaborar la cartera de inversiones en sectores como educación, cultura, saneamiento, salud y transportes, debido a que los proyectos de inversión pública que acarrea efectos positivos sobre la economía, generan puestos de trabajo de los pobladores, lo que les permitirá mejorar su calidad de vida. Además, se podrá tener una población con mayores posibilidades de acceder a bienes y servicios públicos. Considerar inversiones públicas en mayor cuantía debe ir a la par con mejorar la calidad de las mismas, estas deben estar bajo un enfoque por resultados que impacten en las poblaciones más vulnerables.

Se recomienda que en futuras investigaciones se debe considerar el efecto de indicadores de calidad de las inversiones públicas no solo en la pobreza monetaria, sino también de la pobreza con un enfoque multidimensional.

\section{REFERENCIAS}

Alvarado Tolentino, J. (2018). Análisis de la gestión del gasto público en inversión y su incidencia sobre la reducción de los niveles de pobreza en el Perú. Quipukamayoc, 26(51), 33 - 41. Recuperado de https://doi.org/10.15381/quipu.v26i51.14933.

Aparicio, Jaramillo, \& San Román (2011). Desarrollo de la infraestructura y reducción de la pobreza: el caso peruano. (Informe na PB32-2010). Centro de Investigación de la Universidad del Pacífico. https://www.cies.org.pe/sites/default/files/investigaciones/resumen-cies.pdf

Briones, O., \& Peña, E. (2018). Programas sociales y su contribución al desarrollo social Perú 2000 - 2015. Lima, Perú: Universidad Inca Garcilaso de la Vega.

Camones, L. A. (2015). Impacto del gasto en infraestructura productiva en la reducción de la pobreza: Análisis a nivel de gobiernos locales. Lima, Perú. Recuperado de http://tesis.pucp.edu.pe/repositorio/handle/123456789/7242

Fort Meyer, R., \& Paredes Castro, H. (2014). Impacto de la inversión pública rural en el desarrollo de las regiones y de bienestar de la población (2002-2012). Lima: CIES.

Huamaní, Peralta, A. (2016). Inversión pública y sus implicancias en el desarrollo socioeconómico en el Departamento de Puno, Perú. Revista de Investigaciones Altoandinas, 18(3), 337-354. Recuperado de https://dialnet.unirioja.es/servlet/articulo?codigo=5645614

Huaquisto, R. (2018). Inversión pública y pobreza monetaria en el departamento de Puno: periodo 20042015. Tesis. Facultad de Ingeniería Económica. Universidad Nacional del Antiplano. Perú. Recuperado de http://repositorio.unap.edu.pe/bitstream/handle/UNAP/7376/ Huaquisto Ramos River.pdf?sequence $=1 \&$ isAllowed $=y$ 
INEI (2017). Evolución de la Pobreza Monetaria: 2007-2016. Informe Técnico. Lima, Perú. Recuperado de https://www.inei.gob.pe/media/MenuRecursivo/publicaciones digitales/Est/ Lib1533/libro.pdf

Programa de las Naciones Unidas para el Desarrollo (2014). Informe sobre Desarrollo Humano 2014. Nueva York: Programa de las Naciones Unidas para el Desarrollo (PNUD). Recuperado de https://www.undp.org/content/undp/es/home/librarypage/hdr/2014-humandevelopment-report.html

Ponce, S. (2013). Inversión Pública y Desarrollo Económico Regional. Lima, Perú: Pontificia Universidad Católica del Perú.

Recuperado de http://tesis.pucp.edu.pe/repositorio/handle/20.500.12404/4837

Quiñones, M. (2016). Efectos del gasto público sobre la pobreza monetaria en el Perú:2004-2012. Lima, Perú. Recuperado de http://tesis.pucp.edu.pe/repositorio/handle/20.500.12404/7147

Vilca, J. (2018). Inversión pública y su relación con los niveles de pobreza monetaria en las regiones del Perú: periodo 2004-2015. Tesis. Facultad de Ciencias Económicas, Contables y Administrativas. Universidad Nacional de Cajamarca. Perú.

Recuperado de https://repositorio.unc.edu.pe/bitstream/handle/UNC/1887/

T016 45975937 T.pdf?sequence=1\&isAllowed $=y$

Los artículos publicados por IECOS pueden ser compartidos a través de la licencia Creative Commons: CC BY 4.0 Perú. Permisos lejos de este alcance pueden ser consultados a través del correo revistas@uni.edu.pe. 